\title{
Polymers confined between two parallel plane walls
}

\author{
Hsiao-Ping $\mathrm{Hsu}^{\text {a) }}$ and Peter Grassberger ${ }^{\text {b) }}$ \\ John-von-Neumann Institute for Computing, Forschungszentrum Jülich, D-52425 Jülich, Germany
}

(Received 12 September 2003; accepted 31 October 2003)

\begin{abstract}
Single three-dimensional polymers confined to a slab, i.e., to the region between two parallel plane walls, are studied by Monte Carlo simulations. They are described by $N$-step walks on a simple cubic lattice confined to the region $1 \leqslant z \leqslant D$. The simulations cover both regions $D \ll R_{F}$ and $D$ $\gg R_{F}$ (where $R_{F} \sim N^{\nu}$ is the Flory radius, with $\nu \approx 0.587$ ), as well as the cross-over region in between. Chain lengths are up to $N=80000$, slab widths up to $D=120$. In order to test the analysis program and to check for finite size corrections, we actually studied three different models: (a) ordinary random walks (mimicking $\Theta$ polymers); (b) self-avoiding walks; and (c) Domb-Joyce walks with the self-repulsion tuned to the point where finite size corrections for free (unrestricted) chains are minimal. For the simulations we employ the pruned-enriched-Rosenbluth method with Markovian anticipation. In addition to the partition sum (which gives us a direct estimate of the forces exerted onto the walls), we measure the density profiles of monomers and of end points transverse to the slab, and the radial extent of the chain parallel to the walls. All scaling laws and some of the universal amplitude ratios are compared to theoretical predictions. (C) 2004 American Institute of Physics. [DOI: 10.1063/1.1636454]
\end{abstract}

\section{INTRODUCTION}

Although the behavior of flexible polymers in a good solvent confined to different geometries has been studied for many years, ${ }^{1,2}$ there are still a number of open questions. In the present work we shall only discuss single polymer chains between two parallel walls which act only as geometric constraints, without any energetic effects.

Theoretically, this problem is rather well understood. All important scaling laws have been formulated, including the crossover from the region of narrow slabs (where the distance $D$ between the walls is smaller than the Flory diameter of a free coil) to the opposite case of wide slabs. In particular, there exists an important theoretical prediction: Near such a wall the monomer density profile increases as

$$
\rho(z) \sim z^{1 / \nu},
$$

where $z$ is the distance from the wall and $\nu$ is the Flory exponent. ${ }^{1}$ This is supposed to hold for all dimensions (not only for $d=3$ ), and both for ordinary random walks (ideal polymers) for which $\nu=1 / 2$ and for self-avoiding walks with $\nu \approx 0.587$ (in $d=3$ ).

It is intuitively obvious that the force exerted by the polymer onto the wall is proportional to the monomer density near the wall. The ratio between the two can be expressed in terms of a universal amplitude ratio which is easy to calculate for ideal chains, and which was calculated by Eisenriegler $^{3}$ as an expansion in $\epsilon=4-d$. Several authors have tried to verify these detailed predictions by Monte Carlo simulations, ${ }^{4-7}$ but the results are not yet convincing. While the scaling of the density near the wall is roughly

\footnotetext{
${ }^{a)}$ Electronic mail: h.p.hsu@fz-juelich.de

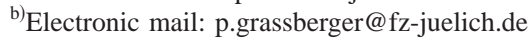

verified, the amplitude ratio consistently has come out too large, casting even doubt on the validity of the $\epsilon$ expansion.

In Ref. 8 we had studied confined polymers in a strip in two dimensions where the amplitude ratio had been predicted by Eisenriegler ${ }^{9}$ (using conformal invariance results of Cardy et al. ${ }^{10}$ ). There we verified all predictions, but we found that this was less easy than anticipated: There are very large corrections to Eq. (1) which can easily be missed, and overlooking them would give wrong estimates of the amplitude ratio. This suggests of course that the same effect was the source of difficulties in $d=3$.

It is the purpose of the present paper to present a careful numerical study, in order to settle these questions. We not only simulated much larger systems than previous authors, going to chain lengths up to $N=80000$, slab widths up to $D=120$, and collecting rather high statistics. Since it is well known that asymptotic scaling of unconstrained selfavoiding walks (SAWs) is reached rather slowly, with correction terms decreasing only as $N^{-0.5},{ }^{11-13}$ we studied also the Domb-Joyce (DJ) model ${ }^{14}$ with $w=0.6$ (where convergence to asymptotia is much faster ${ }^{12,13}$ ) in addition to SAWs.

The DJ model is defined by the partition sum

$$
Z_{N}(w)=\sum_{\text {configs. }} w^{\kappa}
$$

where the sum extends over all random walk (RW) configurations with $N$ steps, $0 \leqslant w \leqslant 1$, and $\kappa$ is the total number of monomer pairs occupying a common site. For $w=1$ the DJ model describes just ordinary random walks. For $w=0$ it is just the SAW model. Asymptotically (for $N \rightarrow \infty$ ) the model is in the SAW universality class for all $w<1$, but the speed with which the renormalization group fixed point is approached depends on $w$. Moreover, it is approached from opposite sides when $w<w^{*}$ and when $w>w^{*}$, with $w^{*}$ 
$\approx 0.6{ }^{12,13}$ For $w=w^{*}$ the approach to asymptotia is fastest, and we thus expect also smaller finite size corrections for the present problem of confined polymers.

Finally, we also performed simulations of the ordinary RW model, just to check the simulation and analysis programs, as everything can be calculated there analytically.

For the simulation we used the pruned-enrichedRosenbluth method (PERM $)^{15}$ with $k$-step Markovian anticipation. ${ }^{8,16-18}$ Apart from being fast (notice that the pivot algorithm which is very fast for unconstrained polymers $^{11}$ is very inefficient for narrow slabs), it has the advantage that the partition sum is computed by default with very high precision. Thus we could estimate the dependence of the monomer fugacity on the width $D$, and from that directly the total forces exerted onto the walls. For more details see Ref. 8.

Details of the scaling predictions are discussed in Sec. II, while results and their comparison with theoretical predictions are presented in Sec. III. Conclusions are finally given in Sec. IV.

\section{SCALING PREDICTIONS}

The end-to-end distance $R_{N}$ of a free SAW in infinite $d$-dimensional volume scales as

$$
R_{N}^{2} \equiv\left\langle\left(\mathbf{x}_{N}-\mathbf{x}_{0}\right)^{2}\right\rangle \approx d(k N)^{2 \nu_{d}}\left(1+b / N^{\Delta_{d}}\right),
$$

where $\nu_{d}$ is the Flory exponent, $\Delta_{d}$ is the leading correction to scaling exponent, and $b$ and $k$ are nonuniversal constants which depend on the microscopic realization. In $d=2$ one has $\nu_{2}=3 / 4,{ }^{1}$ while the best published estimates for $d=3$ are $\nu_{3}=0.5877(6),{ }^{11} 0.5874(2),{ }^{19}$ and $0.58758(7) .{ }^{12}$ In the latter paper it was assumed that the leading correction to Eq. (3) is $1+b / N^{0.5}$. Since this is questionable (our own simulations gave $\Delta_{3} \approx 0.45^{13,20}$ ), we use in the following $\nu_{3}$ $=0.58765(20)$ which also incorporates results from extensive simulations of the DJ model with $w=0.6 .{ }^{20}$ For the DJ model $w^{*}$ is defined by $b\left(w=w^{*}\right)=0$. The absence of large corrections to scaling leads to a rather precise estimate of the constant $k$ in case of the DJ model with $w=0.6: k$ $=0.3259(4),{ }^{20}$ but for 3-d SAWs the estimate is much less stable. The value $k=.4640$ (4) of Ref. 11 depends crucially on the estimate $\Delta \approx 0.56$ made by these authors. Assuming instead $\Delta=0.5$ as in Ref. 12 and the value of $\nu_{3}$ found by these authors, the same data would give $k \approx 0.4655$, while an even larger value would be obtained if $\Delta<0.5$. In the following we shall use $k=0.4657(7)$ for 3-d SAWs.

Equation (3) with $d=2$ also describes the behavior of the parallel components (i.e., parallel to the wall) in the regime $1 \ll D \ll N^{\nu_{3}}$ where the polymer is essentially two dimensional. The constant $k$ depends then on the slab width $D$. A scaling ansatz for the crossover between the two regimes $1 \ll N^{\nu_{3}} \ll D$ and $1 \ll D \ll N^{\nu_{3}}$ is ${ }^{1}$

$$
R_{N, \|}^{2}(D)=R_{N}^{2} \Phi\left(R_{N} / D\right),
$$

where $R_{N}^{2}$ is given by Eq. (3) with $d=3$, and

$$
\Phi(\eta)=\left\{\begin{array}{cc}
2 / 3 & \text { for } \eta \rightarrow 0 \\
\eta^{2\left(\nu_{2} / \nu_{3}-1\right)} & \text { for } \eta \rightarrow \infty
\end{array}\right.
$$

It leads to the prediction ${ }^{6}$

$$
k(D) \sim D^{\left(\nu_{3}-\nu_{2}\right) / \nu_{2} \nu_{3}} .
$$

The partition sum of a free SAW in infinite volume scales for $N \rightarrow \infty$ as

$$
Z_{N}=\mu_{\infty}^{-N} N^{\gamma_{d}-1} \text { const }
$$

with $\mu_{\infty}$ being the critical fugacity per monomer, and with $\gamma_{d}$ being a universal exponent. In two dimensions $\gamma_{2}$ $=43 / 32,{ }^{1}$ while the best published estimate for $d=3$ is $\gamma_{3}$ $=1.1575(6){ }^{21}$ In the following we shall use the estimate $\gamma_{3}=1.1575$ (3) obtained from the DJ model with $w=0.6 .^{20}$ For 3-d SAWs one has $\mu_{\infty}=0.213491(4)$ from exact enumerations $^{22}$ and $\mu_{\infty}=0.2134910$ (3) from Monte Carlo simulations. ${ }^{13,20}$ In the following we shall use the latter. For the DJ model we use $\mu_{\infty}=0.18812145(7) .{ }^{20}$

Again we must expect that the same ansatz, with $\gamma_{3}$ replaced by $\gamma_{2}$, with $\mu_{\infty}$ replaced by $\mu(D)$, and with the constant replaced by $c(D)$, holds for slabs in the limit $D$ $\ll N^{\nu_{3}}$ :

$$
Z_{N}(D)=\mu(D)^{-N} N^{\gamma_{2}-1} c(D) \text { for } D \ll N^{\nu_{3}} \text {. }
$$

The corresponding crossover ansatz is then

$$
Z_{N}(D)=Z_{N} \Psi\left(R_{N} / D\right)
$$

with

$\Psi(\eta)$

$$
=\left\{\begin{array}{cc}
\text { const } & \text { for } \eta \rightarrow 0 \\
\eta^{\left(\gamma_{2}-\gamma_{3}\right) / \nu_{3}} \exp \left(-\frac{a}{\mu_{\infty} k}(\eta / \sqrt{3})^{1 / \nu_{3}}\right) & \text { for } \eta \rightarrow \infty,
\end{array}\right.
$$

$$
\mu(D)=\mu_{\infty}+a D^{-1 / \nu_{3}}
$$

and

$$
c(D)=\operatorname{const} D^{\left(\gamma_{2}-\gamma_{3}\right) / \nu_{3}} .
$$

Notice in particular that the $D$ dependence of $\mu(D)$, Eq. (11), follows directly from the scaling ansatz for the crossover. We should also point out that the partition function for a polymer in a slab is defined as the sum over all walks starting at fixed $\mathbf{x}_{0, \|}=\left(x_{0}, y_{0}\right)$, but averaged over all $z_{0}$ $\in[1, D]$.

The force exerted onto the wall is most straightforwardly expressed in terms of the work done when moving one of the walls, i.e., by the dependence of the free energy-and thus also of the partition sum-on $D$,

$$
F=k_{B} T \frac{d \ln Z_{N}(D)}{d D},
$$

where we have introduced a dummy temperature $T$ which can take any positive value. From Eqs. (8) and (11), the force per monomer is then obtained as

$$
f=F / N=k_{B} T \frac{a}{\nu_{3} \mu_{\infty}} D^{-1-1 / \nu_{3}}
$$

in the limit of $D \rightarrow \infty$ and $N \gg D^{1 / \nu_{3}}$.

The monomer density near the wall is predicted to scale with Eq. (1). For ordinary random walks this gives $\rho(z)$ $\sim z^{2}$, but in that case one can compute $\rho(z)$ exactly, with the result 


$$
D \rho(z)=1-\cos (2 \pi z / D) \equiv f(z / D)
$$

for $D \gg 1$ [we normalize $\rho(z)$ such that $\sum_{z=1}^{D} \rho(z)=1$ ]. For walks with excluded volume $\rho(z)$ is not known, but one expects $D \rho(z)$ to be universal.

One should expect that the density near the walls is proportional to the force per monomer. Indeed it was shown by Eisenriegler $^{3}$ that

$$
\lim _{z \rightarrow 0} k \frac{\rho(z)}{z^{1 / \nu_{3}}}=B \frac{f}{k_{B} T}=B \frac{a}{\nu_{3} \mu_{\infty}} D^{-1-1 / \nu_{3}}
$$

with $B$ being a universal amplitude ratio. For ideal chains one has $B=2$, while for chains with excluded volume in 4 $-\epsilon$ dimensions one has $B \approx 2\left(1-b_{1} \epsilon\right)$ with $b_{1}=0.075 .^{3}$ In three dimensions this gives the prediction $B \approx 1.85$.

Finally, the density profile of end points scales near the walls with a new exponent which is related to the surface exponent in spin systems. ${ }^{23}$ For polymers, the standard way to introduce this exponent is via the partition sum of a SAW, one end of which is glued to an impenetrable wall. For this system one has

$$
Z_{N}^{(1)} \sim \mu_{\infty}^{-N} N^{\gamma_{d}^{(1)}-1}
$$

with $\gamma_{3}^{(1)}=0.679(2){ }^{24}$ The end point density then scales like $^{25}$

$$
\rho_{\text {end }}(z) \sim z^{\left(\gamma-\gamma^{(1)}\right) / \nu} \sim z^{0.814(6)},
$$

where the right-hand side holds for $d=3$.

\section{RESULTS}

\section{A. Random walks}

In order to test our simulation and analysis methods, we first simulate the simple model of RWs on a simple cubic lattice between two hard walls at $z=0$ and $z=D+1$, confining the polymer to $1 \leqslant z \leqslant D$. We simulated width $D$ up to 80 and chain length between 3500 (for $D=4$ ) and 80000 (for $D=80)$.

Monomer densities are shown in Fig. 1. They were obtained by averaging over the central part of the chains, excluding $10 \%$ on either side to avoid errors from the fact that Eq. (1) should hold only far away from the ends, for monomer indices $n$ satisfying $D^{2} \ll n \ll N-D^{2}$ (we should mention that $N / D^{2}>10$ for all data sets). For finite $D$ the scaling has to be slightly modified, by replacing in Eq. (15) $z / D$ by $\xi$ $=z /(D+1)$ and $D \rho(z)$ by $(D+1) \rho(z)$. We see that all data, even for small $D$, fall precisely onto the predicted curve. To show that also the regions near the walls are correctly sampled, we plot in panel (b) of Fig. 1 the same data but divided by the product of the two power laws for $z \approx 0$ and $z \approx D,(D+1) \rho(z) / f_{0}(\xi)$ with $f_{0}(\xi)=2 \pi^{2}[\xi(1-\xi)]^{2}$.

Critical fugacities are determined by plotting $Z_{N} \mu_{D}^{N}$ against $\log N$ and demanding that these curves become horizontal for large $N$. Results are shown in Fig. 2, where we plot $\mu_{D}-\mu_{\infty}$ with $\mu_{\infty}=1 / 6$. The dashed line is not a fit to the data, but fits their extrapolation to $D \rightarrow \infty, \mu_{D}-\mu_{\infty}$ $=0.2741 /(D+1)^{2}$. This agrees with Eq. (11) (since $\nu$ $=1 / 2$ ) and gives $a=0.2741(2)$, where the error is obtained by assuming that the slope is 2 as predicted. On the other
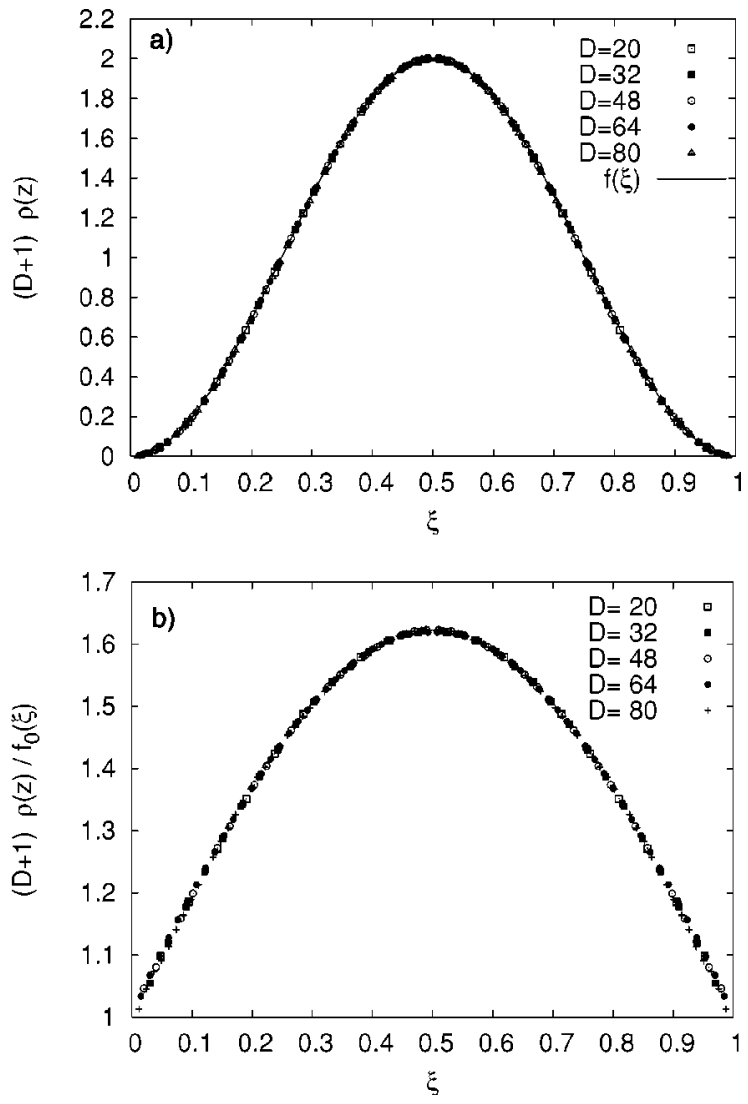

FIG. 1. Rescaled values of the monomer density, $(D+1) \rho(z)$ against $\xi$ $=z /(D+1)$ for ordinary random walks. Also plotted is the function $f(\xi)$ $=2 \sin ^{2}(\pi \xi)$. (b) The same values, but divided by $f_{0}(\xi)=2 \pi^{2}[\xi(1-\xi)]^{2}$.

hand, Eqs. (16) and (15) together with $B=2, k=1 / 3$, and $\mu_{\infty}=1 / 6$ give $a=\pi^{2} / 36=0.27416$, in perfect agreement. We should point out that Eq. (11) is significantly violated for small $D$ in this model, and becomes exact only for large $D$. We shall see the same behavior also for SAWs and for the DJ model, and we had seen the same also in $d=2 .^{8}$

In Fig. 3 we show the transverse distribution of chain ends. For ordinary RWs it should be just the square root of the monomer density, i.e., proportional to $\sin (\pi \xi)$. This is obviously true for our data, with high precision.

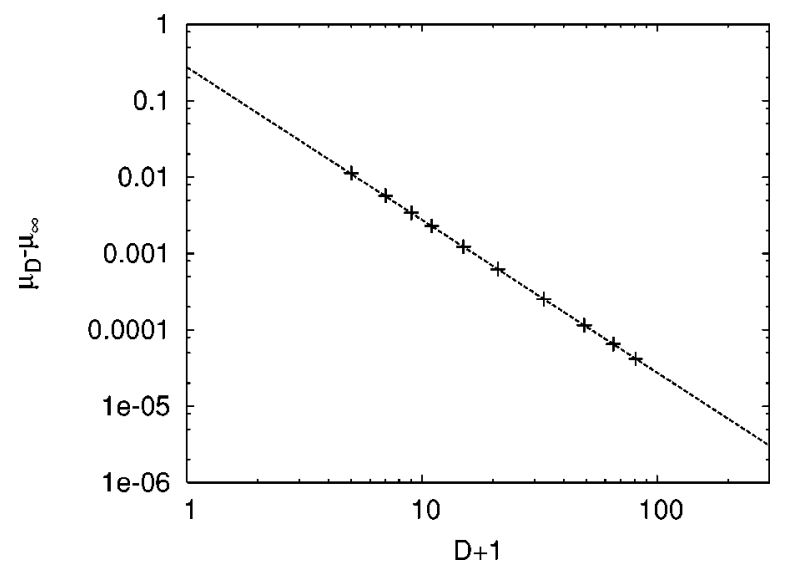

FIG. 2. $\log -\log$ plot of $\mu_{D}-\mu_{\infty}$ against $D+1$, for ordinary random walks. The dashed line is $\mu_{D}-\mu_{\infty}=0.2741 /(D+1)^{2}$ and gives our best extrapolation of the data for large $D$. 


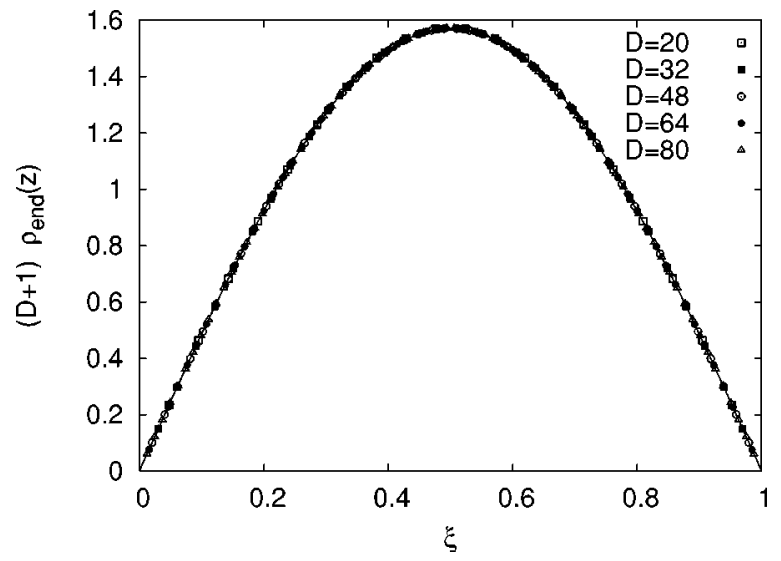

FIG. 3. Rescaled values of the probability $\rho_{\text {end }}(z)$ that the chain end is at the distance $z$ from a wall against $\xi=z /(D+1)$, for ordinary random walks. The solid line is the function $(\pi / 2) \sin (\xi)$.

Finally we should mention that the crossover ansatzes, Eqs. (4) and (9), become trivial for RWs, since the critical exponents are the same in $d=2$ and $d=3$. We therefore also do not show data for $R_{N, \|}$ which can be calculated approximately by assuming that all steps are uncorrelated, and that vertical steps occur with probability $1 / 3$ inside the slab and with probability $1 / 5$ at the boundaries.

\section{B. Self-avoiding walks}

Let us first discuss the trivial case $D=1$. In this case we have ordinary 2-d SAWs, and therefore we can use the comparison with the known results as a test for our algorithm. Our simulations, with $N=3000$, gave indeed perfect agreement for the critical exponents, and also the amplitude for the end-to-end distance, $R_{\|}^{2} / N^{2 \nu_{2}} \approx 0.771(1)$, in agreement with the value obtained in Ref. 11 .

In our nontrivial simulations we used widths up to $D$ $=120$ and chain length up to $N=80000$. As first tests we checked the crossover ansatzes Eqs. (4) and (9). In these tests we replaced $R_{N}$ and $Z_{N}$ by parametrizations similar to Eqs. (4) and (9), but including additional correction to scaling terms. As seen from Figs. 4 and 5, the data collapse is ex-

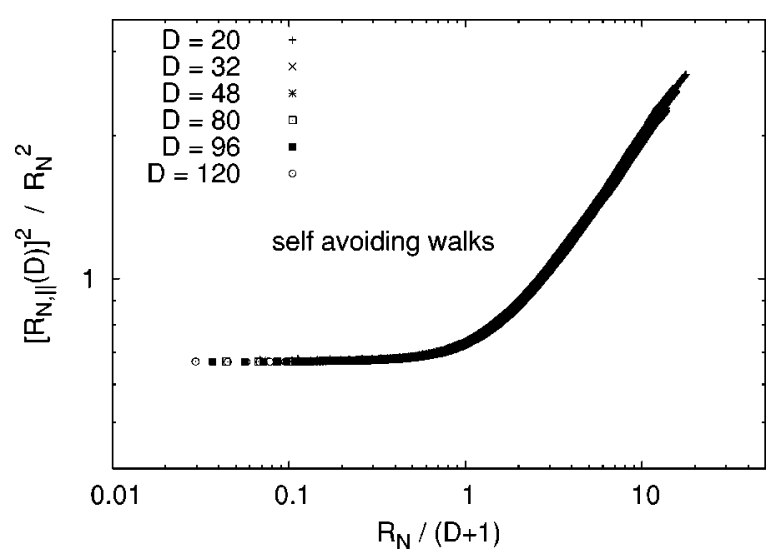

FIG. 4. Data collapse for testing the crossover ansatz Eq. (4) for selfavoiding walks.

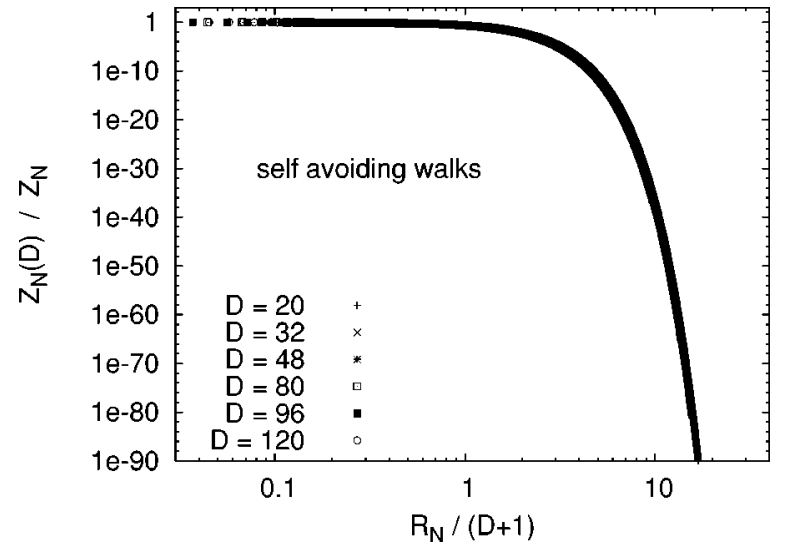

FIG. 5. Data collapse for testing the crossover ansatz Eq. (9) for self avoiding walks.

cellent. Such a perfect collapse would not have been obtained, if we had replaced $R_{N}$ and $Z_{N}$ by the leading asymptotic powers of $N .^{26}$

Critical fugacities were determined by plotting $\log Z_{N}$ $-\left(\gamma_{2}-1\right) \log N+N x$ against $N$ and changing $x$ until these curves become horizontal for large $N$. Then $\mu(D)=\exp (x)$. Results are shown in Fig. 6, where we plot $\mu(D)-\mu_{\infty}$ against $D$. As for ordinary RWs, the plot does not give a straight line (replacing $D$ by $D+1$ would improve the situation a bit, but not much), so the straight line shown in Fig. 6 indicates the estimated asymptotic behavior, assuming its slope to be given by $-1 / \nu_{3}$. It provides us with the estimate $a=0.448 \pm 0.005$.

The monomer densities for different values of $D$, again from the central region only, are shown in Fig. 7. Plotting the densities directly as in panel (a) indicates that scaling is satisfied. But it is not very informative, since deviations from scaling in the important regions near the walls would not show up. Also, panel (a) might suggest that $\rho(z)$ is simply the product of two powers,

$\rho(z) \approx \frac{1}{D+1} f_{0}\left(\frac{z}{D+1}\right) \quad$ with $\quad f_{0}(\xi)=A[\xi(1-\xi)]^{1 / \nu_{3}}$,

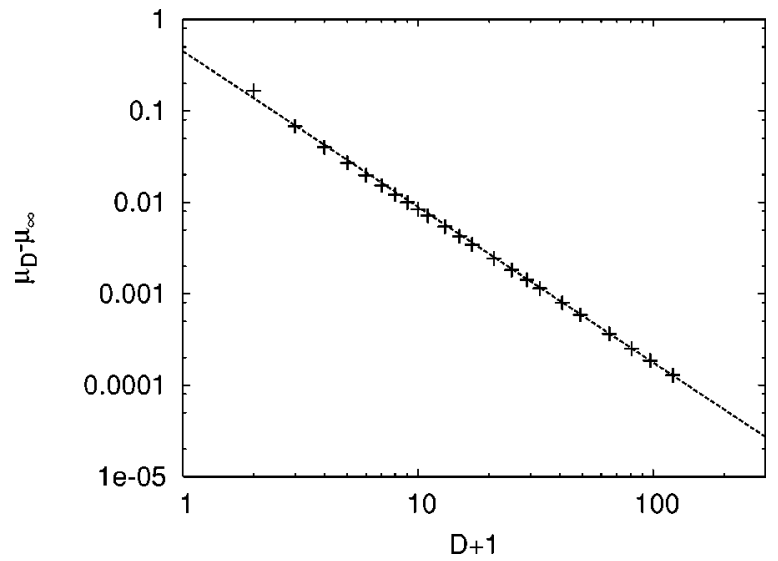

FIG. 6. $\log -\log$ plot of $\mu_{D}-\mu_{\infty}$ against $D+1$. The dashed line is $\mu_{D}$ $-\mu_{\infty}=0.448(D+1)^{-1 / \nu_{3}}$. 

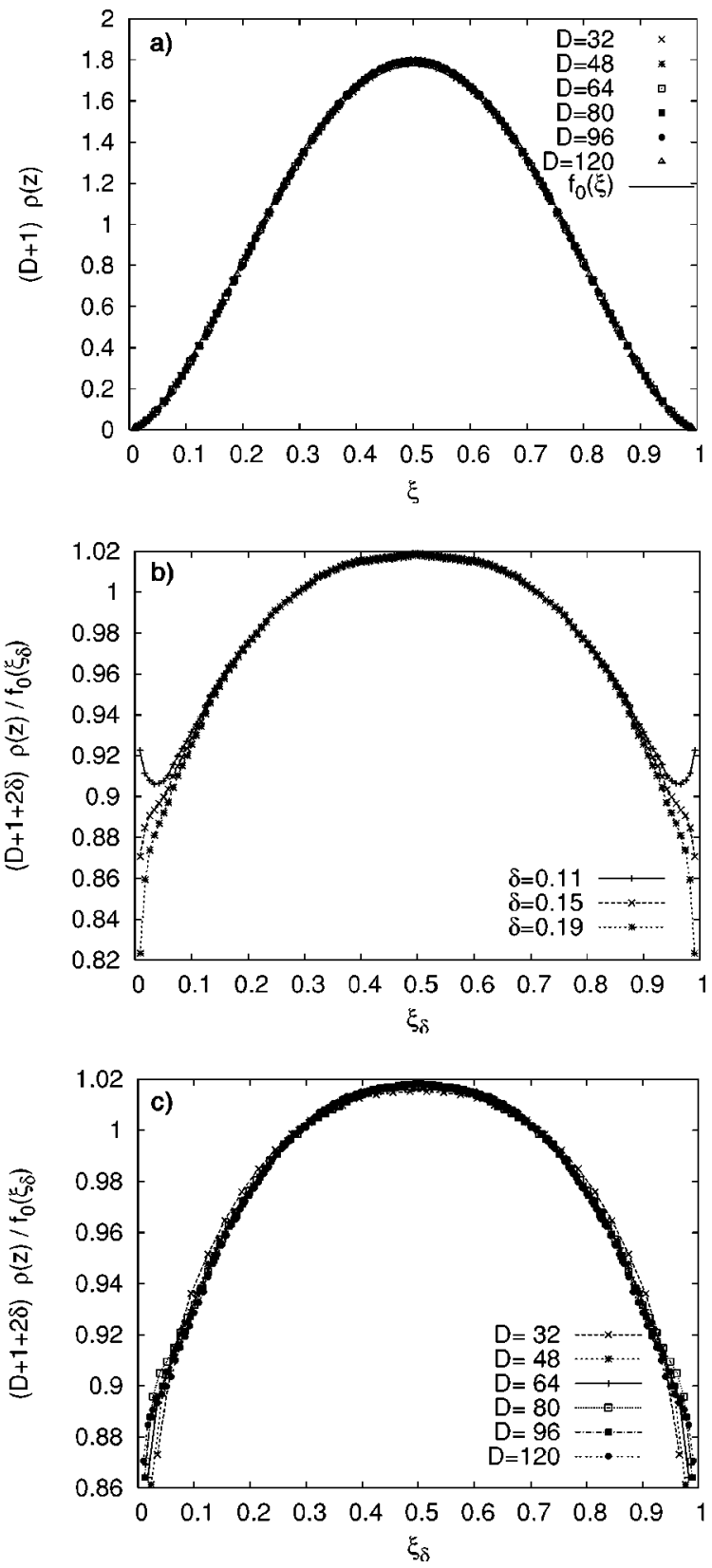

FIG. 7. (a) Rescaled values of the monomer density $(D+1) \rho(z)$ against $\xi=z /(D+1)$. Also plotted is the function $f_{0}(\xi)=18.74(\xi(1-\xi))^{1 / \nu_{3}}$. (b) The data for $D=120$ plotted against a modified scaling variable, $\xi_{\delta}=(z$ $+\delta) /(D+1+2 \delta)$, and divided by $f_{0}\left(\xi_{\delta}\right)$, for three different values of $\delta$. (c) The data for all $D$, again divided by $f_{0}\left(\xi_{\delta}\right)$ with $\delta=0.15$.

where the constant $A=18.74$ is determined by normalization. We had already seen for random walks that $\rho(z)$ is not that simple, and indeed plotting $(D+1) \rho(z) / f_{0}(z /(D+1))$ as in panels (b) and (c) shows that this would be a very bad approximation. In addition, panel (b) shows that one has to introduce an "extrapolation length" $\delta$ as suggested in Ref. 6 so that the scaling variable $\xi$ is replaced by

$$
\xi_{\delta}=\frac{z+\delta}{D+1+2 \delta} .
$$

Best scaling near $z=0$ and $z=D$ [panel (b)] and best data collapse [panel (c)] is obtained for $\delta \approx 0.15$, although a closer inspection of these figures shows that neither the scal-

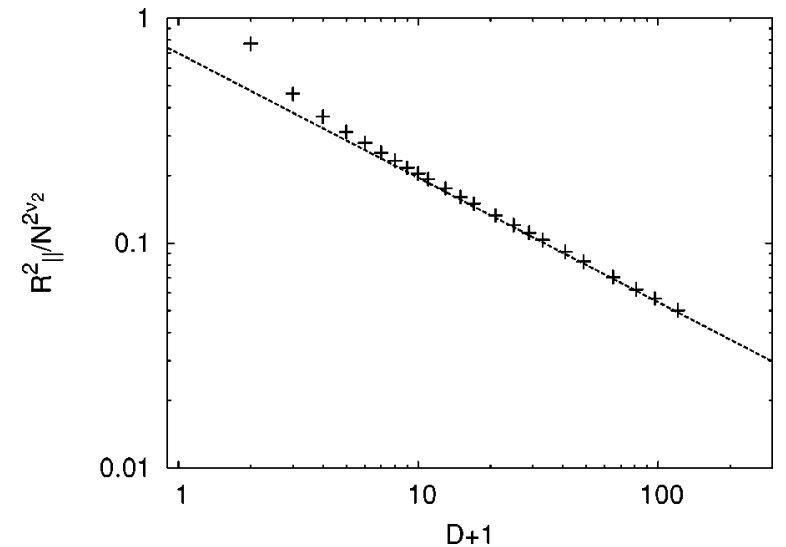

FIG. 8. $\log -\log$ plots of $R_{\|}^{2}(D) / N^{2 \nu_{2}}$ vs $D$. The dashed line is 0.697 ( $D$ $+1)^{-0.553}$.

ing nor the data collapse are perfect. These small persistent discrepancies and the overestimation of the amplitude $B$ discussed in the next paragraph were the main reasons for studying the Domb-Joyce model.

Figure $7(\mathrm{c})$ suggests that $D^{1+1 / \nu_{3}} \rho(z) / z^{1 / \nu_{3}} \rightarrow 0.87(4)$ $\times A=16.1(8)$ for $z \rightarrow 0$ and $D \rightarrow \infty$. The very large uncertainty reflects the rather steep slopes at $z=0$ and $z=D$. Using this in Eq. (16) gives $B=2.13 \pm 0.11$. This is larger than the prediction of Eisenriegler, ${ }^{3}$ but much less so than previous estimates. ${ }^{6,7}$ We believe that these previous authors had missed the fact that $\rho(z) / f_{0}(\xi)$ is not constant. If we would assume $\rho(z) \propto f_{0}(\xi)$, we would obtain $B \approx 2.48$, which is indeed similar to the previous Monte Carlo estimates.

As a further test of scaling we checked in detail that $R_{\|}(D) \sim N^{\nu_{2}}$ for $N^{\nu_{2}} \gg D$, and we estimated the asymptotic ratios between the two. They are plotted in Fig. 8, where we also plotted the scaling prediction,

$$
R_{\|}(D)^{2} / N^{2 \nu_{2}} \sim D^{-2\left(\nu_{2}-\nu_{3}\right) / \nu_{3}}=D^{-0.553} .
$$

Finally, we show in Fig. 9 the distribution $\rho_{\text {end }}(z)$ of chain ends. We found that $\rho_{\text {end }}(z)$ is very closely proportional to $\left(\xi_{\delta}\left(1-\xi_{\delta}\right)\right)^{0.865}$ with $\delta=0.3$, but the (very small) deviations are highly significant. Taking them into account, we find

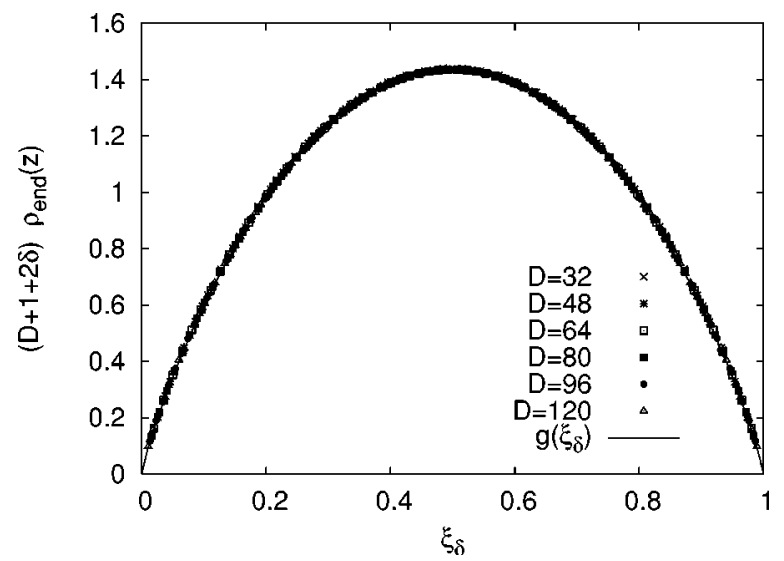

FIG. 9. Rescaled values of the probability $\rho_{\text {end }}(z)$ that the chain end is at the distance $z$ from a wall, against $\xi_{\delta}=(z+\delta) /(D+1+2 \delta)$ with $\delta=0.3$. The solid line is the function $g\left(\xi_{\delta}\right)=4.78\left(\xi_{\delta}\left(1-\xi_{\delta}\right)\right)^{0.865}$. 


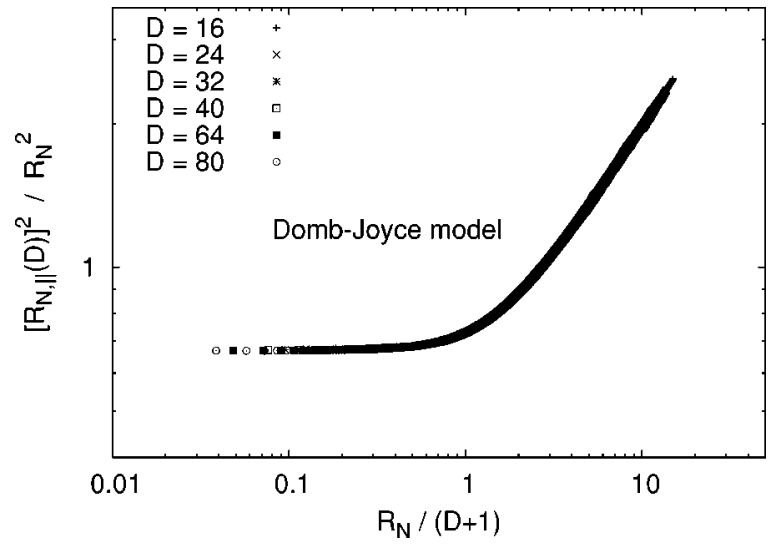

FIG. 10. Data collapse for testing the crossover ansatz Eq. (4) for DombJoyce walks.

$$
\rho_{\text {end }}(z) \sim z^{0.80(2)}
$$

near the walls, with $\delta \approx 0.2$. This agrees nicely with Eq. (18).

\section{Domb-Joyce model}

Domb-Joyce chains with interaction strength $w=0.6$, which is very close to critical strength $w^{*}$ where leading corrections to scaling vanish, ${ }^{12,13}$ were studied for slab widths up to $D=80$. Chain lengths were up to $N=72000$. The analysis of the data was done exactly as for the selfavoiding walks described in Sec. III B.

As expected, the scaling functions $\Phi(\eta)$ and $\Psi(\eta)$ are very similar to those for SAWs (see Figs. 10 and 11). This universality verifies that the amplitudes and critical exponents discussed in Sec. II are essentially correct, although this should not be taken too seriously: Such data collapse plots are not very sensitive to details (look at the huge range of scales in Fig. 11!).

Estimates of the critical fugacities are shown in Fig. 12, where we plot $\mu_{D}-\mu_{\infty}$ against $D+1$. The straight line, which again represents the extrapolation to large $D$, provides the estimate $a=0.2813(6)$.

Plots of the monomer density profile (Fig. 13) are very similar to those for SAWs. But the extrapolation length is now much smaller, $\delta \approx 0.04$ as compared to $\delta \approx 0.15$ for

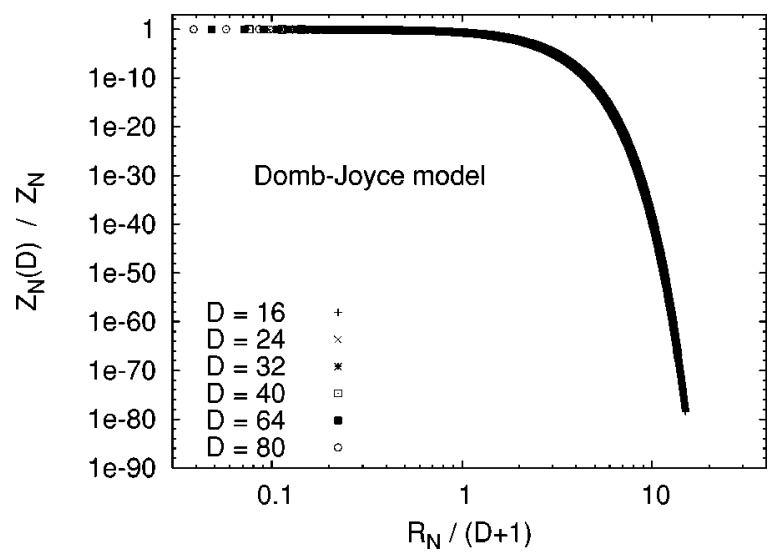

FIG. 11. Data collapse for testing the crossover ansatz Eq. (9) for DombJoyce walks.

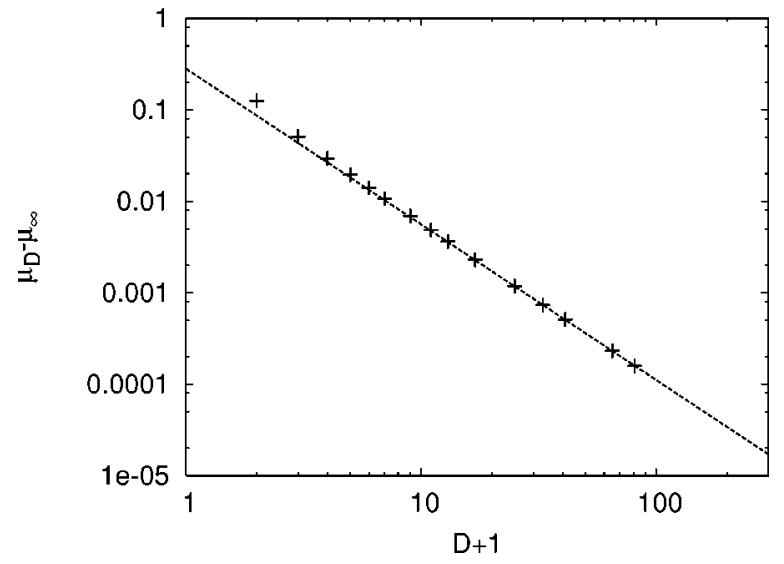

FIG. 12. $\log -\log$ plot of $\mu_{D}-\mu_{\infty}$ against $D+1$ for the Domb-Joyce model. The dashed line is $\mu_{D}-\mu_{\infty}=0.2813(D+1)^{-1 / \nu_{3}}$ with $\nu_{3}$ used as constraint.

SAWs. This is a first indication that corrections to scaling are indeed smaller in the DJ model. More important, also the scaling curve in Fig. 13(c) looks slightly different from that in Fig. 7(b): It is considerably smaller at the walls, with $\lim _{z \rightarrow 0, D \rightarrow \infty} D^{1+1 / \nu_{3}} z^{-1 / \nu_{3}} \rho(z) / A=0.71(3)$ as compared to 0.87(4) for SAWs. Given the fact that scaling corrections should be smaller for the DJ model (in spite of the somewhat smaller values of $D$ ), we consider the DJ value as more correct, and blame the discrepancy onto scaling corrections
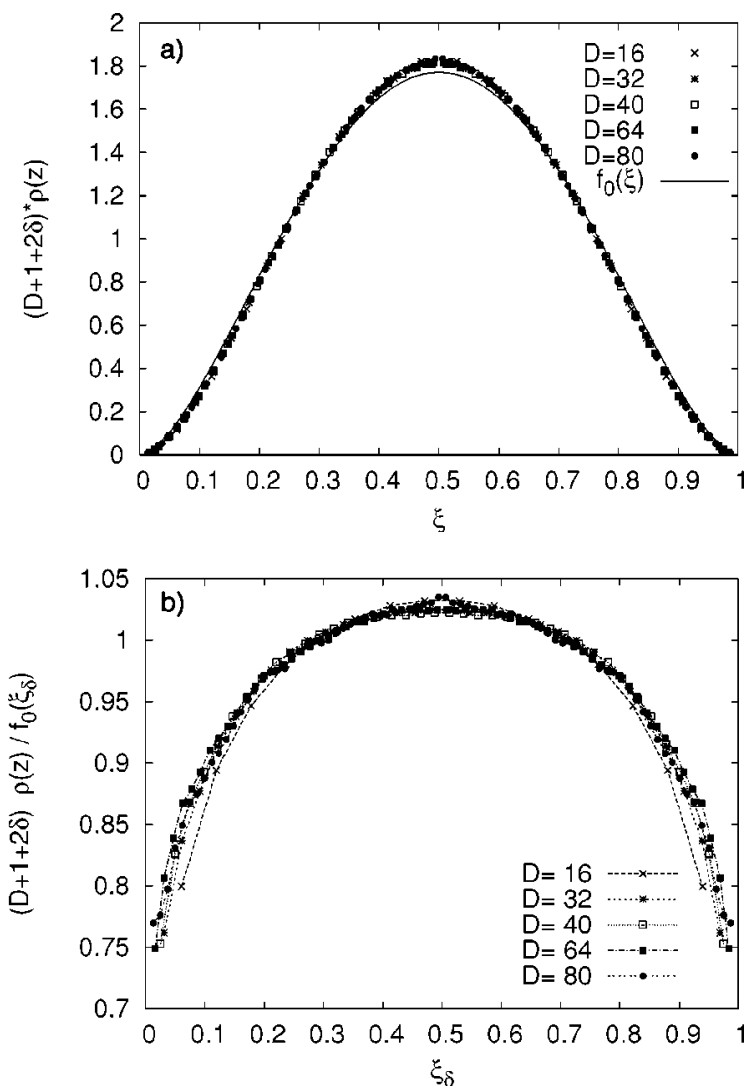

FIG. 13. (a) Rescaled values of the monomer density $(D+1+2 \delta) \rho(z)$ of the Domb-Joyce model against $\xi=(z+\delta) /(D+1+2 \delta)$ with $\delta=0.06$. Also plotted is the function $f_{0}(\xi)=18.74(\xi(1-\xi))^{1 / \nu_{3}}$. (b) The same values as in (a), but divided by $f_{0}\left(\xi_{\delta}\right)$ with $\delta=0.04$. 


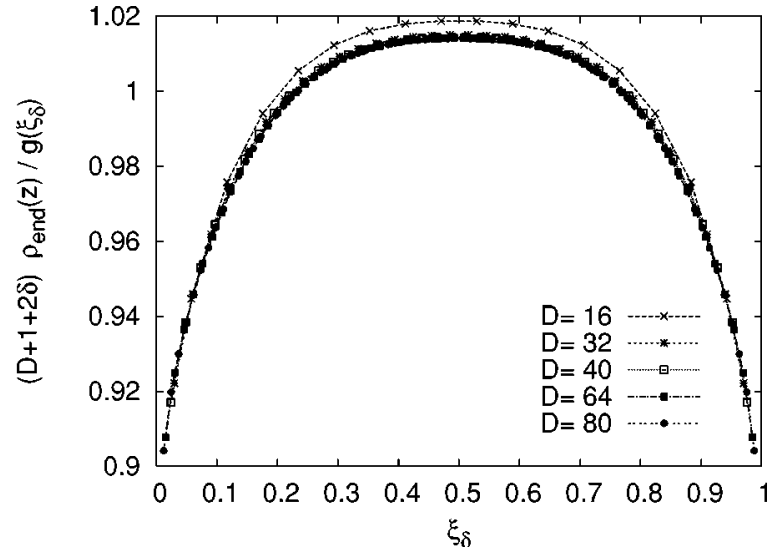

FIG. 14. Rescaled values of the probability $\rho_{\text {end }}(z)$ that the chain end is at the distance $z$ from a wall, divided by the function $g\left(\xi_{\delta}\right)=4.358\left(\xi_{\delta}(1\right.$ $\left.-\xi_{\delta}\right)^{0.81}$, against $\xi_{\delta}=(z+\delta) /(D+1+2 \delta)$ with $\delta=-0.02$.

for SAWs. With this new estimate of $\lim _{z \rightarrow 0, D \rightarrow \infty} D \rho(z)$, and using the nonuniversal amplitude $a$ determined earlier, we obtain our final estimate for the universal amplitude ratio $B$,

$$
B=1.70 \pm 0.08 \text {. }
$$

This is only 2 standard deviations away from the $\epsilon$ prediction $B=1.85$ of Eisenriegler, ${ }^{3}$ which we consider as good agreement.

Finally, we do not show our data for $R_{N, \|}(D) / N^{\nu_{2}}$ and for the end monomer profile, since they are very similar to Figs. 8 and 9. But again the seemingly perfect agreement of the end point density profile is again as deceptive as it was for SAWs. This time our best estimate for the scaling of the end point distribution is

$$
\rho_{\text {end }}(z) \sim z^{0.81(1)},
$$

with $\delta \approx-0.02$, in even better agreement with Eq. (18) than the estimate for SAWs. The rescaled density profile divided by $g\left(\xi_{\delta}\right)=4.358\left(\xi_{\delta}\left(1-\xi_{\delta}\right)\right)^{0.81}$ is shown in Fig. 14 .

\section{SUMMARY}

We have presented high statistics simulations of 3-d polymers, modeled as walks on a simple cubic lattice with either hard or soft excluded volume interactions, with chain length up to 80000 on slabs of widths up to 120 . This was possible with the PERM algorithm with Markovian anticipation. The fact that PERM gives by default very precise estimates of free energies allowed us to measure precisely the forces exerted onto the walls, by measuring how the critical fugacities depend on the width of the slabs. We verified all critical scaling laws predicted for this problem, including the scaling of monomer and end point densities near the walls and the scaling of the total pressure with chain length and with slab width.

The theoretical prediction most difficult to verify numerically concerns the amplitude ratio between the pressure onto the wall and the monomer density close to the wall. Previous simulations had not been able to obtain this with sufficient precision, and also in the present paper we had serious problems when using self-avoiding walks with strict (hard-core) self repulsion. This might not be so surprising, given the well-known fact that SAWs show rather large corrections to scaling. These corrections to scaling can be minimized by going over to Domb-Joyce polymers (characterized by soft repulsion) with carefully adjusted strength of the repulsion (similarly, for off-lattice bead-spring models, one can adjust the ratio between bead size and equilibrium spring length to minimize corrections to scaling). It was only when going over to this Domb-Joyce model that we could verify in detail all theoretical predictions.

Thus we have shown, first of all, that already the field theoretic $\epsilon$ expansion to first order in $\epsilon$, as implemented in Ref. 3, gives correct results. This was not obvious, in particular in view of persistent previous difficulties to verify it by Monte Carlo simulations. Second, we have demonstrated again the importance of using models with minimized corrections to scaling. And last but not least we have again shown that recursive sequential sampling methods with resampling $^{29}$ (of which PERM is a particular implementation) can be very efficient.

\section{ACKNOWLEDGMENTS}

We thank Professor Erich Eisenriegler and Professor Ted Burkhardt for valuable discussions, and Dr. Walter Nadler for carefully reading the manuscript.

${ }^{1}$ P. G. de Gennes, Scaling Concepts in Polymer Physics (Cornell University Press, Ithaca, NY, 1979).

${ }^{2}$ E. Eisenriegler, Polymers Near Surfaces (World Scientific, Singapore, 1993).

${ }^{3}$ E. Eisenriegler, Phys. Rev. E 55, 3116 (1997).

${ }^{4}$ I. Webman, J. L. Lebowitz, and M. H. Kalos, J. Phys. (Paris) 41, 579 (1980).

${ }^{5}$ T. Ishinabe, J. Chem. Phys. 83, 423 (1985).

${ }^{6}$ A. Milchev and K. Binder, Eur. Phys. J. B 3, 477 (1998); 13, 607 (2000).

${ }^{7}$ J. De Joannis, J. Jimenez, R. Rajagopalan, and I. Bitsanis, Europhys. Lett. 51, 41 (2000).

${ }^{8}$ H.-P. Hsu and P. Grassberger, Eur. Phys. J. B (to be published); e-print cond-mat/0308276 (2003).

${ }^{9}$ E. Eisenriegler, in Field Theoretical Tools in Polymer and Particle Physics, edited by H. Meyer-Ortmanns and A. Klümper (Springer, Heidelberg, 1997).

${ }^{10}$ J. Cardy and G. Mussardo, Nucl. Phys. B 410, 451 (1993).

${ }^{11}$ B. Li, N. Madras, and A. D. Sokal, J. Stat. Phys. 80, 661 (1995).

${ }^{12}$ P. Belohorec and B. G. Nickel, Guelph University, 1997 (preprint).

${ }^{13}$ P. Grassberger, P. Sutter, and L. Schäfer, J. Phys. A 30, 7039 (1997).

${ }^{14}$ C. Domb and G. S. Joyce, J. Phys. C 5, 956 (1972).

${ }^{15}$ P. Grassberger, Phys. Rev. E 56, 3682 (1997).

${ }^{16}$ H. Frauenkron, P. Grassberger, and N. Walter, e-print cond-mat/9806321 (1998).

${ }^{17}$ H. Frauenkron, M. S. Causo, and P. Grassberger, Phys. Rev. E 59, R16 (1999).

${ }^{18}$ S. Caracciolo, M. S. Causo, P. Grassberger, and A. Pelissetto, J. Phys. A 32, 2931 (1999).

${ }^{19}$ T. Prellberg, J. Phys. A 34, L599 (2001).

${ }^{20} \mathrm{P}$. Grassberger (unpublished).

${ }^{21}$ S. Caracciolo, M. S. Causo, and A. Pellisetto, Phys. Rev. E 57, 1215 (1998).

${ }^{22}$ D. MacDonald, S. Joseph, D. L. Hunter, L. L. Moseley, N. Jan, and A. J. Guttmann, J. Phys. A 33, 5973 (2000).

${ }^{23} \mathrm{H}$. W. Diehl, in Phase Transitions and Critical Phenomena edited by C. Domb and J. L. Lebowitz (Academic, New York, 1986), Vol. 10.

${ }^{24}$ P. Grassberger and R. Hegger, J. Phys. A 27, 4069 (1994).

${ }^{25}$ E. Eisenriegler, K. Kremer, and K. Binder, J. Chem. Phys. 77, 6296 (1982).

${ }^{26}$ While $R_{N, \|}^{2}(D)$ increases monotonically with $1 / D$, this is not true for the 
full three-dimensional $R_{N}^{2}(D)$, since the compression in the $z$ direction overwhelms the swelling in $x$ and $y$, for large and intermediate values of $D$ (Refs. 27 and 28).

${ }^{27}$ J. H. van Vliet and G. Brinke, J. Chem. Phys. 93, 1436 (1990); J. H. van
Vliet, M. C. Luiken, and G. Brinke, Macromolecules 25, 3802 (1992).

${ }^{28}$ C. E. Cordeiro, M. Molisana, and D. Thirumalai, J. Phys. II 7, 433 (1997).

${ }^{29}$ J. S. Liu, Monte Carlo Strategies in Scientific Computing, Springer Series in Statistics (Springer, New York, 2001). 